\title{
Determining Day of Given Date Mathematically
}

\author{
R. Sivaraman \\ National Awardee for Popularizing Mathematics among Masses, D G Vaishnav College, India
}

Received July 14, 2020; Revised August 20, 2020; Accepted September 17, 2020

\section{Cite This Paper in the following Citation Styles}

(a): [1] R. Sivaraman, "Determining Day of Given Date Mathematically," Mathematics and Statistics, Vol. 8, No. 5, pp. 590 - 595, 2020. DOI: 10.13189/ms.2020.080514.

(b): R. Sivaraman (2020). Determining Day of Given Date Mathematically. Mathematics and Statistics, 8(5), 590 - 595. DOI: $10.13189 / \mathrm{ms} .2020 .080514$.

Copyright $\mathrm{C} 2020$ by authors, all rights reserved. Authors agree that this article remains permanently open access under the terms of the Creative Commons Attribution License 4.0 International License

\begin{abstract}
Computation of day of a week from given date belonging to any century has been a great quest among astronomers and mathematicians for long time. In recent centuries, thanks to efforts of some great mathematicians we now know methods of accomplishing this task. In doing so, people have developed various methods, some of which are very concise and compact but not much accessible explanation is provided. The chief purpose of this paper is to address this issue. Also, almost all known calculations involve either usage of tables or some pre-determined codes usually assigned for months, years or centuries. In this paper, I had established the mathematical proof of determining the day of any given date which is applicable for any number of years even to the time of BCE. I had provided the detailed mathematical derivation of month codes which were key factors in determining the day of any given date. Though the procedures for determining the day of given date are quite well known, the way in which they arrived is not so well known. This paper will throw great detail in that aspect. To be precise, I had explained the formula obtained by German Mathematician Zeller in detail and tried to simplify it further which will reduce its complexity and at the same time, would be as effective as the original formula. The explanations for Leap Years and other astronomical facts were clearly presented in this paper to aid the derivation of the compact form of Zeller's Formula. Some special cases and illustrations are provided wherever necessary to clarify the computations for better understanding of the concepts.
\end{abstract}

Keywords Leap Years, Congruence, Modulo Arithmetic, Ceiling Function, Floor Function, Centurial Years, Month Codes

\section{Introduction}

Ever since humans came to understand the functioning of universe for various reasons, various forms of calendars have been put in to use. Different civilizations used different calendars. Since October 1582, when Pope Gregory XIII introduced a new calendar as a correction to then existing Julian Calendar, various parts of the globe at different times, adopted the new calendar. At present, the calendar introduced by Pope Gregory XIII was followed throughout the globe and it was named in his honour as "Gregorian Calendar".

Before we start our actual mathematical investigation, we glance through the basic structure of Gregorian calendar system.

The Gregorian calendar is a solar calendar with 12 months of 28-31 days each. A regular Gregorian year consists of 365 days, but in certain years known as leap years, a leap day is added to February. Gregorian years are identified by consecutive year numbers. Various countries had their own beginning of the year until in recent centuries everyone had accepted the fact that January 1 as the beginning of a New Year. Thus, the present Gregorian calendar consider one year from $1^{\text {st }}$ January to $31^{\text {st }}$ December of any year containing 12 months and 365/366 days.

In ancient times, astronomers knew only about seven planets. Probably this might be the reason for fixing seven days for a week. Hence, all the calendar calculations regarding determination of day of a given date were based on the number 7. Thanks to various mathematicians and astronomers we now have a global calendar satisfying our needs. 


\section{Leap Years}

In the Gregorian calendar, the orbital period of the Earth around the Sun is not 365 days, but closer to 365.2425 days. To account for this longer period, every four years, we add an extra day (since $0.25 \times 4=1$ ) in February, to make 366 days.

But observe that $365.25-365.2425=0.0075$. So adding 1 day in February for every 4 years will produce an over estimate of $0.0075 \times 4=3$ days. To bring down these 3 days, it is suggested that the years like $1600,1700,1800$, $1900,2000,2100, \ldots$ (called centurial years) which are divisible by 4 , would be considered as leap years if they are not only divisible by 4 but also by 400 . This arrangement ensures us to count only 1 leap year and leaves 3 years among any consecutive 400 years period like $1301-1600$, $1601-2000,2001-2400$, etc.

Thus, among the period of 400 years from $1601-2000$, the years $1700,1800,1900$ were not leap years though they are divisible by 4 but not by 400 , whereas, 2000 is a leap year since it is divisible by both 4 and 400 . In this viewpoint, we present the following rule for a year being a Leap Year (which will be crucial for our task of finding day of given date):

"Every year that is exactly divisible by 4 is a leap year, except for years that are exactly divisible by 100 , but these centurial years are leap years if they are exactly divisible by 400."

As a consequence of the above rule, we see that, in the century from $1601-1700$, there are 24 leap years (as 1700 is not a leap year). Similarly, the centuries $1701-1800$, 1801 - 1900 each contain 24 leap years (since 1800, 1900 are not leap years) but the century $1901-2000$ contain 25 leap years as 2000 is a leap year. Hence in every period of four consecutive centurial years, there will be $(24 \times 3)+25$ $=97$ Leap years for every 400 years in Gregorian calendar.

\section{Cyclic Property of Gregorian Calendar}

In a period of every 400 years like say $1701-2000$ or $2001-2400, \ldots$ we will find the total number of days. As there are 97 leap years in every 400 years the total number of days would be given by $(365 \times 303)+(366 \times 97)=$ $110595+35502=146097$.

Since $\frac{146097}{7}=20871$, the total number of days in every 400 years namely 146097 is exactly divisible by 7 , it follows that there are exactly 20871 weeks in a period of every 400 years. Hence the whole system of Gregorian calendar years repeats for every 400 years. This phenomenon can be termed as "Cyclic Property" of Gregorian calendar.

Due to this, we see that the calendar for the year 1582 (in which modern Gregorian calendar) was introduced will be same as the years 1982, 2382, 2782, ... Similarly, the calendars for the years $1487,1887,2287,2687 . \ldots$ are identical.

Note that when we apply Cyclic Property rule for years occurring in BCE time, we should add 1 to 400 and make it 401 , since there is no year 0 between $1 \mathrm{BCE}$ and $1 \mathrm{CE}$. Thus, the calendar for the year 44 BCE in which Julius Ceasar was assassinated would be same as $-44+401=$ $357 \mathrm{CE}$ which would be same as for the years 757,1157 , $1557,1957,2357, \ldots$

\section{Known Formula for Determining Day of Given Date}

Exactly 300 years after Gregorian calendar got introduced; the problem of finding day of a given date in compact computable form was studied by German mathematician Julius Christian Johannes Zeller, who published an elegant algorithm for the same in 1882. Now this algorithm was named after him as "Zeller's Congruence".

According to Zeller's Congruence rule, the day of any date of the form $d / m / Y(d$ - date, $m$ - month, $Y$-Year) is given by

$$
\left(d+\left\lfloor\frac{13(m+1)}{5}\right\rfloor+Y+\left\lfloor\frac{Y}{4}\right\rfloor-\left\lfloor\frac{Y}{100}\right\rfloor+\left\lfloor\frac{Y}{400}\right\rfloor\right)(\bmod 7) \rightarrow(1)
$$

Here, $\lfloor x\rfloor$ is called the Floor Function or Greatest Integer Function of $x$.

$\lfloor x\rfloor$ is defined to be greatest integer $\leq x$. As a consequence of this definition, we find that if $x>0$ such that $x=a \cdot d_{1} d_{2} d_{3} d_{4} \cdots$ then $\left\lfloor a \cdot d_{1} d_{2} d_{3} d_{4} \cdots\right\rfloor=a$. Similarly, if $x<0$ such that $a \geq 0$ and if $x=-a \cdot d_{1} d_{2} d_{3} d_{4} \cdots$ then $\left\lfloor-a \cdot d_{1} d_{2} d_{3} d_{4} \cdots\right\rfloor=a-1$.

For example, $\lfloor\sqrt{2}\rfloor=1,\left\lfloor\frac{\pi}{4}\right\rfloor=0,\left\lfloor\frac{-\pi}{2}\right\rfloor=-2$.

Similarly, $(\bmod 7)$ denote the remainder when the whole term inside the bracket is divided upon by 7 .

\section{Explanation of Zeller's Congruence Rule}

Let us first rewrite the Zeller's Congruence Formula and try to understand it in a better perspective.

$$
\begin{gathered}
\left(d+\left\lfloor\frac{13(m+1)}{5}\right\rfloor+Y+\left\lfloor\frac{Y}{4}\right\rfloor-\left\lfloor\frac{Y}{100}\right\rfloor+\left\lfloor\frac{Y}{400}\right\rfloor\right)(\bmod 7) \rightarrow(1) \\
3 \leq m \leq 14
\end{gathered}
$$

For a given date of the form $d / m / Y$, where $d, m, Y$ represents the date, month and year respectively, we note that Zeller's Formula in (1) contain 6 terms inside the bracket. The first and third terms $d$ and $Y$ are included as it 
is for computation.

Using the Leap year rule mentioned in 2., we get the fourth, fifth and sixth terms given by

$$
\left\lfloor\frac{Y}{4}\right\rfloor-\left\lfloor\frac{Y}{100}\right\rfloor+\left\lfloor\frac{Y}{400}\right\rfloor
$$

The second term regarding month $m$ need little explanation.

When making these calculations, Zeller made a novel approach by beginning the year with the month of March (instead of January) and ending with February of next subsequent year. With this assumption, the twelve months of a year in formula (1), is considered from $m=3,4,5, \ldots, 14$ where $m=3$ corresponding to March, $m=4$ for April and so on until $m=12$ for December, $m=13$ for January and $m$ $=14$ for February but Zeller took $Y-1$ instead of $Y$ for the months January and February.

Thus for example, for calculating day corresponding to any date of January 2020, according to Zeller's formula we should consider $m=13$ and $Y=2019$ (As beginning with March of 2019, January 2020 is viewed as $13^{\text {th }}$ month of 2019). Similarly, for finding any date corresponding to February 2020, we should consider $m=14$ since this is considered as $14^{\text {th }}$ and last month of the year 2019. This explains the reason for the term $m=3,4,5, \ldots, 14$ associated with the formula (1).

Considering the shift of year beginning from January to March, the number of days in each month is as follows: $\{31$, $30,31,30,31,31,30,31,30,31,31,28 / 29\}$ corresponding to the number of days from months March to February. Since any week contain seven days we shall modulo 7 (that is, divide each number by 7 and take the remainders) giving $\{3,2,3,2,3,3,2,3,2,3,3,0\}$.

If we now cumulative sum of five consecutive numbers from the above list we get $3+2+3+2+3=13,2+3+2$ $+3+3=13,3+2+3+3+2=13$, and so on. In general, any set of five consecutive numbers from the above modulo list of numbers will constitute three 3's and two 2's always giving a sum of 13 . So for every 5 numbers we get a sum of 13. This explains the reason for the term 13/5 in the second term of formula (1). Since any week day should be one of seven days, we finally divide the answer obtained through 6 terms of the formula by 7 and take out the remainder explaining the term $(\bmod 7)$ in the formula.

We know that when any integer is divided by 7 , the possible remainders are $0,1,2,3,4,5,6$. Now depending upon the remainder, we shall consider the following assignment of days corresponding to these remainders to decide the day of a given date.

0 - Saturday, 1 - Sunday, 2 - Monday, 3 - Tuesday, 4 - Wednesday, 5 - Thursday, 6 - Friday.

\section{Sample Computations}

(i) Let us determine the day of $1 / 1 / 1$ the first day of Common Era denoted by CE.
By cyclic property of Gregorian calendar, we can find the day for $1 / 1 / 401$ instead of 1/1/1. Here $d=1, m=13, Y=$ 400 (Since it is January) Zeller's Congruence yields the following value:

$$
\begin{aligned}
& \left(1+\left\lfloor\frac{13 \times(13+1)}{5}\right\rfloor+400+\left\lfloor\frac{400}{4}\right\rfloor-\left\lfloor\frac{400}{100}\right\rfloor\right. \\
& \left.+\left\lfloor\frac{400}{400}\right\rfloor\right)(\bmod 7) \\
& =(1+36+400+100-4 \\
& +1)(\bmod 7)=2
\end{aligned}
$$

Since 2 correspond to Monday, it follows that the first day of Common Era (CE) is a Monday.

(ii) Let us now find on what day does the most famous physicist of $20^{\text {th }}$ Century Albert Einstein was born? It is known that he was born on $14^{\text {th }}$ March (Incidentally, it was now celebrated as world Pi Day) of 1879. Thus we have $d=14, m=3, Y=1879$. Using Zeller's formula we get:

$$
\begin{gathered}
\left(14+\left\lfloor\frac{13 \times(3+1)}{5}\right\rfloor+1879+\left\lfloor\frac{1879}{4}\right\rfloor-\left\lfloor\frac{1879}{100}\right\rfloor\right. \\
\left.+\left\lfloor\frac{1879}{400}\right\rfloor\right)(\bmod 7)=6
\end{gathered}
$$

Since 6 correspond to Friday, we know that Albert Einstein was born on Friday.

Similar to these calculations, it is possible for us to determine the day of any given date. Note that if the date correspond to BCE, then add 401 and convert it in to $\mathrm{CE}$, then proceed in the same way as presented above. Now that I have explained the formation and application of the Zeller's Congruence Formula, I will present ways to reduce the complexity of the formula presented above and also derive the month codes which will be used for several centuries at the same time thanks to Cyclic property of Gregorian calendar.

\section{Rewriting Zeller's Formula}

We see that if the year number $Y$ is quite large as in the case for Einstein date, it is usually difficult to perform the calculation in our mind. We can reduce the size of $Y$ suitably and make Zeller's original formula in much more compact way. In this part, I provide mathematical proofs for doing that.

If $Y=100 c+y$, where $c$ denote the first two digits of $Y$ and $y$ denote the last two digits of $Y$. Note here that $y$ varies from 0 to 99 (both 0 and 99 inclusive). With this assumption, we will calculate the third, fourth, fifth and sixth terms involved in Zeller's Formula.

(i) Reduction of Third Term:

$$
Y(\bmod 7)=(100 c+y)(\bmod 7) \equiv(2 c+y)(\bmod 7)
$$


(ii) Reduction of Fourth Term

$$
\begin{gathered}
\left\lfloor\frac{Y}{4}\right\rfloor(\bmod 7)=\left\lfloor\frac{100 c+y}{4}\right\rfloor(\bmod 7) \\
\quad=\left\lfloor 25 c+\frac{y}{4}\right\rfloor(\bmod 7) \\
\quad \equiv\left(4 c+\left\lfloor\frac{y}{4}\right\rfloor\right)(\bmod 7)
\end{gathered}
$$

(iii) Reduction of Fifth Term

$$
\begin{aligned}
& \left\lfloor\frac{Y}{100}\right\rfloor(\bmod 7)=\left\lfloor\frac{100 c+y}{100}\right\rfloor(\bmod 7) \\
& \quad=\left\lfloor c+\frac{y}{100}\right\rfloor(\bmod 7) \\
& \quad \equiv(c+0)(\bmod 7)=c(\bmod 7)
\end{aligned}
$$

(iv) Reduction of Sixth Term

$$
\begin{aligned}
& \left\lfloor\frac{Y}{400}\right\rfloor(\bmod 7)=\left\lfloor\frac{100 c+y}{400}\right\rfloor(\bmod 7) \\
& =\left\lfloor\frac{c}{4}+\frac{y}{100}\right\rfloor(\bmod 7) \equiv\left\lfloor\frac{c}{4}\right\rfloor(\bmod 7)
\end{aligned}
$$

Substituting these in the original Zeller's formula we get

$$
\begin{aligned}
\left(Y+\left\lfloor\frac{Y}{4}\right\rfloor-\left\lfloor\frac{Y}{100}\right\rfloor+\left\lfloor\frac{Y}{400}\right\rfloor\right)(\bmod 7) \\
=\left((2 c+y)+4 c+\left\lfloor\frac{y}{4}\right\rfloor-c\right. \\
\left.+\left\lfloor\frac{c}{4}\right\rfloor\right)(\bmod 7) \\
=\left(y+\left\lfloor\frac{y}{4}\right\rfloor+\left\lfloor\frac{c}{4}\right\rfloor+5 c\right)(\bmod 7) \\
\equiv\left(y+\left\lfloor\frac{y}{4}\right\rfloor+\left\lfloor\frac{c}{4}\right\rfloor-2 c\right)(\bmod 7)
\end{aligned}
$$

Thus the original Zeller's formula now becomes

$$
\left(d+\left\lfloor\frac{13(m+1)}{5}\right\rfloor+y+\left\lfloor\frac{y}{4}\right\rfloor+\left\lfloor\frac{c}{4}\right\rfloor-2 c\right)(\bmod 7) \rightarrow(2)
$$

Equation (2) is a concise formula to find the day of any given date compared to the calculation involved through Equation (1). Moreover, Equation (2) is the usual reference of Zeller's formula in many sources for which we have obtained a mathematical derivation. We shall call Equation (2) as modified Zeller's Formula.

\section{Derivation of Month Codes}

In 7., we saw the reduction of Year number $Y$ considerably with lesser numbers $c, y$. Here we use Equation (2) to derive month codes which will further reduce our calculation of finding day of any given date.

First, let us substitute each value of $m$ from 3 to 14 (from March to February) successively in the second term ofZeller's formula which contains the information about months.

$$
m=3 \rightarrow\left\lfloor\frac{13(m+1)}{5}\right\rfloor(\bmod 7)=\left\lfloor\frac{13(3+1)}{5}\right\rfloor(\bmod 7) \equiv 3
$$

(March)

$$
\begin{aligned}
& m=4 \rightarrow\left\lfloor\frac{13(m+1)}{5}\right\rfloor(\bmod 7)=\left\lfloor\frac{13(4+1)}{5}\right\rfloor(\bmod 7) \equiv 6 \quad(\text { April }) \\
& m=5 \rightarrow\left\lfloor\frac{13(m+1)}{5}\right\rfloor(\bmod 7)=\left\lfloor\frac{13(5+1)}{5}\right\rfloor(\bmod 7) \equiv 1 \\
& m=6 \rightarrow\left\lfloor\frac{13(m+1)}{5}\right\rfloor(\bmod 7)=\left\lfloor\frac{13(6+1)}{5}\right\rfloor(\bmod 7) \equiv 4 \\
& m=7 \rightarrow\left\lfloor\frac{13(m+1)}{5}\right\rfloor(\bmod 7)=\left\lfloor\frac{13(7+1)}{5}\right\rfloor(\bmod 7) \equiv 6 \\
& m=8 \rightarrow\left\lfloor\frac{13(m+1)}{5}\right\rfloor(\bmod 7)=\left\lfloor\frac{13(8+1)}{5}\right\rfloor(\bmod 7) \equiv 2 \\
& m=9 \rightarrow\left\lfloor\frac{13(m+1)}{5}\right\rfloor(\bmod 7)=\left\lfloor\frac{13(9+1)}{5}\right\rfloor(\bmod 7) \equiv 5
\end{aligned}
$$

(September)

$$
\begin{aligned}
& m=10 \rightarrow\left\lfloor\frac{13(m+1)}{5}\right\rfloor(\bmod 7)=\left\lfloor\frac{13(10+1)}{5}\right\rfloor(\bmod 7) \equiv 0 \\
& m=11 \rightarrow\left\lfloor\frac{13(m+1)}{5}\right\rfloor(\bmod 7)=\left\lfloor\frac{13(11+1)}{5}\right\rfloor(\bmod 7) \equiv 3
\end{aligned}
$$

(November)

$$
m=12 \rightarrow\left\lfloor\frac{13(m+1)}{5}\right\rfloor(\bmod 7)=\left\lfloor\frac{13(12+1)}{5}\right\rfloor(\bmod 7) \equiv 5
$$

(December)

Since January and February months are considered for the previous year, we have to subtract 1 from the original second term to get correct codes for these months. Thus, we obtain

$$
\begin{aligned}
& m=13 \rightarrow\left\lfloor\frac{13(m+1)}{5}-1\right\rfloor(\bmod 7)=\left\lfloor\frac{13(14+1)}{5}-1\right\rfloor(\bmod 7) \equiv 0 \\
& (\operatorname{January}) \\
& m=14 \rightarrow\left\lfloor\frac{13(m+1)}{5}-1\right\rfloor(\bmod 7)=\left\lfloor\frac{13(14+1)}{5}-1\right\rfloor(\bmod 7) \equiv 3
\end{aligned}
$$

(February)

Thus the month codes beginning from January to December would be the following numbers respectively:

$$
0,3,3,6,1,4,6,2,5,0,3,5 \rightarrow(3)
$$

Now, keeping the Cyclic Property in mind, we first segregate the centuries for every 400 years in to four classes as

Class I: $(1-100,401-500,801-900,1201-1300$, $1601-1700,2001-2100,2401-2500,2801-2900, \quad \ldots)$

Class II: $(101-200,501-600,901-1000,1301-1400$, $1701-1800,2101-2200,2501-2600,2901-3000, \ldots$ )

Class III: $(201-300,601-700,1001-1100,1401-$ 
1500, $1801-1900,2201-2300,2601-2700,3001-$ $3100, \ldots)$

Class IV: $(301-400,701-800,1101-1200,1501-$ $1600,1901-2000,2301-2400,2701-2800,3101-$ $3200, \ldots)$

Note that according to the cyclic property, the days of each century in the corresponding class would be same. Hence, I try to derive the month codes each for the above four classes which will eventually cover all centuries.

Now considering current century years which is from 2001 to 2100 beginning to Class I of our segregation, we find that $c=20$. With this value of $c$, we try to compute fifth and sixth terms of the modified Zeller's formula given in Equation (2).

$$
c=20 \rightarrow\left(\left\lfloor\frac{c}{4}\right\rfloor-2 c\right)(\bmod 7)=\left(\left\lfloor\frac{20}{4}\right\rfloor-2 \times 20\right)(\bmod 7) \equiv 0
$$

(Class I).

Similarly considering the century from $1701-1800$ belonging to Class II, we get $c=17$. Doing as above, we obtain

$$
c=17 \rightarrow\left(\left\lfloor\frac{c}{4}\right\rfloor-2 c\right)(\bmod 7)=\left(\left\lfloor\frac{17}{4}\right\rfloor-2 \times 17\right)(\bmod 7) \equiv 5
$$

(Class II)

Similarly considering $1801-1900$ corresponding to Class III and $1901-2000$ of Class IV we obtain

$$
\begin{aligned}
& c=18 \rightarrow\left(\left\lfloor\frac{c}{4}\right\rfloor-2 c\right)(\bmod 7)=\left(\left\lfloor\frac{18}{4}\right\rfloor-2 \times 18\right)(\bmod 7) \equiv 3 \\
& c=19 \rightarrow\left(\left\lfloor\frac{c}{4}\right\rfloor-2 c\right)(\bmod 7)=\left(\left\lfloor\frac{19}{4}\right\rfloor-2 \times 19\right)(\bmod 7) \equiv 1
\end{aligned}
$$

(Class IV)

The final answers namely $0,5,3,1$ for centuries belonging to corresponding classes will fix the month codes required for easy computation of the day of a given date.

Since for Class I, the answer is 0 , the month codes for any century belonging to Class I are given by precisely Equation (3) namely: 0, 3, 3, 6, 1, 4, 6, 2, 5, 0, 3, 5.

Now since the answer for Class II is 5 , the month codes for any century belonging to Class II are obtained by just adding (modulo 7) to each of the month codes of Class I given above.

Thus the month codes for Class II centuries are: 5, 1, 1, 4, $6,2,4,0,3,5,1,3$.

In similar fashion, by adding 3 and 1 respectively to month codes of Class I, we get month codes of each century belonging to Class III and Class IV.

The month codes for Class III centuries are: $3,6,6,5,4$, $0,2,5,3,3,6,1$

The month codes for Class IV centuries are: $1,4,4,0,2$, $5,0,3,6,1,4,6$

We thus summarize the month codes of each Class for quick reference.

Class I : $0,3,3,6,1,4,6,2,5,0,3,5$.

Class II : $5,1,1,4,6,2,4,0,3,5,1,3$ (Class I codes +

5) $(\bmod 7)$

Class III : 3, 6, 6, 5, 4, 0, 2, 5, 3, 3, 6, 1 (Class I codes

$+3)(\bmod 7)$

Class IV : 1, 4, 4, 0, 2, 5, 0, 3, 6, 1, 4, 6 (Class I codes +1$)(\bmod 7)$

\section{Remarks:}

Note that upon applying these month codes to find day of a given date, we should follow the three rules given below:

(i) Subtract 1 from the month codes corresponding to January/February of normal leap years which are divisible by 4 except the centurial leap years like 400 , $800,1200,1600,2000,2400, \ldots$

(ii) Subtract 2 from the month codes corresponding to any month for all centurial non-leap years like 100, 200, $300,500,600,700,900,1000,1100,1300, \ldots$

(iii) Subtract 1 for all months from March to December and 2 for January/February for centurial leap years like $400,800,1200,1600,2000, \ldots$.

\section{Simplification of Actual Formula}

Using these month codes, we can greatly simplify the modified Zeller's Formulas given in Equation (2). If we do so, we get the following compact formula:

$\left(d+M+y+\left\lfloor\frac{y}{4}\right\rfloor\right)(\bmod 7) \rightarrow(4)$ where $M$ is the month code for the century belonging to one of the four classes mentioned above.

The formula described in Equation (4) is usually presented in many books and online sources for determining day of a given date. I have just provided the mathematical proof for arriving that result.

We now consider three illustrations to justify the formula described by Equation (4).

(i) Let us consider SrinivasaRamanujan's Birthdate which is on $22 / 12 / 1887$. First we notice that the year 1887 lies in Class III. Hence $M=1$ (the month code for December in Class III). Also $d=22, y=87$. Hence by Equation (4) we have

$$
\left(22+1+87+\left\lfloor\frac{87}{4}\right\rfloor\right)(\bmod 7) \equiv 5 \cdot \text { Since } 5 \text { correspond to }
$$

Thursday, we can conclude that one of the greatest mathematicians of India, Srinivasa Ramanujan was born on Thursday.

(ii) Let us consider the Birthdate of another famous living great Indian mathematician C.S. Seshadri who was born on 29/2/1932. We notice that 1932 correspond to Class IV and 1932 is a normal leap year. Hence, $M=4$ $-1=3$ (month code of February -1 ) according to rule (i) presented in the Remarks presented above. Also, $d$ 
$=29, y=32$. Hence by Equation (4), we have $\left(29+3+32+\left\lfloor\frac{32}{4}\right\rfloor\right)(\bmod 7) \equiv 2$. Since, 2 correspond to Monday, we know that C.S. Seshadri was born on Monday, but unfortunately he could celebrate his birthday once in four years only.

(iii) Let us now consider the date 18/8/1900 the Birthdate of Smt. Vijayalakshmi Pandit, sister of Jawaharlal Nehru, the first Prime Minister of India. Note that 1900 is a centurial year and it belongs to $8^{\text {th }}$ month of Class III. Hence $M=5-2=3$ (month code of August -2$)$. Here, $d=18, y=0$. Hence by Equation (4) we have $(18+3+0+0)(\bmod 7) \equiv 0$. Since, 0 correspond to Saturday, we can conclude that Smt. Vijayalakshmi Pandit was born on Saturday.

Thus depending on a normal year, normal leap year or centurial year knowing the class in which the given date belong, we can immediately compute the day quite easily without using any tools.

\section{Proving an Important Fact}

Using Equation (4) along with the rules presented in Remarks, we can prove the following interesting but important calendar fact mathematically.

\section{Theorem:}

"The last day of a century cannot be a Tuesday, Thursday or Saturday"

\section{Proof:}

Because of the cyclic property, it is enough to consider the dates $31 / 12 / 1700,31 / 12 / 1800,31 / 12 / 1900$ and $31 / 12 / 2000$ as these dates correspond to date of end of $17^{\text {th }}$, $18^{\text {th }}, 19^{\text {th }}$ and $20^{\text {th }}$ centuries. Since $y=0$ for all these four dates, the third and fourth terms in Equation (4) namely $\left(y+\left\lfloor\frac{y}{4}\right\rfloor\right\rfloor$ becomes 0 . We also note that $d=31$ for all these four dates. Since $31 \equiv 3(\bmod 7)$, from Equation $(4)$, we see that the day corresponding to these four dates would be of the form $(3+M)(\bmod 7)$, where $M$ is the month code corresponding to December month of respective centuries.

We know that the years 1700, 1800, 1900 and 2000 correspond to classes I, II, III and IV respectively. Now for the three centurial non - leap years 1700, 1800, 1900 we need to subtract 2 from the actual month codes given by their respective classes. In doing so, we get $M=5-2=3$ (for 1700), $M=3-2=1$ (for 1800), $M=1-2=-1 \equiv 6(\bmod 7)($ for 1900$)$.

For the centurial leap year 2000, according to rule (iii) in Remarks of 8 , since the month is a December, we should subtract 1 from the actual to get $M=6-1=5$. Using these values of $M$, we can determine the days for the required four dates as follows:

$$
\begin{aligned}
& 31 / 12 / 1700 \rightarrow(3+3)(\bmod 7)=6 \text { which is on Friday. } \\
& 31 / 12 / 1800 \rightarrow(3+1)(\bmod 7)=4 \text { which is on }
\end{aligned}
$$

Wednesday

$31 / 12 / 1900 \rightarrow(3+6)(\bmod 7)=2$ which is on Monday

$31 / 12 / 2000 \rightarrow(3+5)(\bmod 7) \equiv 1$ which is on Sunday

Thus by cyclic property of every 400 years in Gregorian calendar, we see that the end of a century cannot occur on Tuesday or Thursday or Saturday.

\section{Conclusions}

The aspects of making Gregorian calendar and process of arriving the formulas and month codes were explained mathematically in this paper. With little practice, one can easily determine the day of any given date without having to seek any source from electronic equipments.

\section{REFERENCES}

[1] Zeller, Christian, "Die Grundaufgaben der Kalenderrechnung auf neue und vereinfachte Weise gelöst". WürttembergischeVierteljahrsheftefürLandesgeschichte (in German), Issue V, pp. 313-314, 1882.

[2] Zeller, Christian, "Kalender-Formeln". Mathematisch-natur wissenschaftlicheMitteilungendes mathematisch-naturwiss enschaftlichenVereins in Württemberg (in German). Volume 1 (1): pp. 54-58, 1885.

[3] V. F. Rickey, "Mathematics of the Gregorian calendar", Math. Intelligencer, Volume 7, pp. 53-56, 1985.

[4] J. Dutka, "On the Gregorian revision of the Julian calendar", Math. Intelligencer, Volume 10, pp. 56-64, 1988.

[5] G. Moyer, “The Gregorian calendar', Sci. Amer., Vol. 246, Issue 5, pp. 144-152, May 1982.

[6] W. M. Feldman, "Rabbinical Mathematics and Astronomy", M. L. Cailingold, London, 1931; 3rd corrected ed., Sepher-Hermon, New York, 1978.

[7] E. M.'Reingold, J. Nievergelt and N. Deo, “Combinatorial Algorithms: Theory and Practice", Prentice-Hall, Englewood Cliffs, NJ, 1977.

[8] J. V. Uspensky and M. A. Heaslet, "Elementary Number Theory", McGraw-Hill, New York, 1939.

[9] Black Paul E., NIST document, "Zeller's Congruence", Dictionary of Algorithms and Data Structures, Webpage.

[10] Webpage of Data Genetics blog, Zeller's Congurence" at $\mathrm{http}: / /$ datagenetics.com/blog/november12019/index.html 